\title{
Philosophiques
}

\section{Réponses au commentaire de M. André Gombay sur La structure performative du langage juridique}

\section{Georges-A. Legault}

Volume 5, numéro 2, octobre 1978

URI : https://id.erudit.org/iderudit/203103ar

DOI : https://doi.org/10.7202/203103ar

Aller au sommaire du numéro

Éditeur(s)

Société de philosophie du Québec

ISSN

0316-2923 (imprimé)

1492-1391 (numérique)

Découvrir la revue

Citer ce document

Legault, G.-A. (1978). Réponses au commentaire de M. André Gombay sur $L a$ structure performative du langage juridique. Philosophiques, 5(2), 317-329. https://doi.org/10.7202/203103ar d'utilisation que vous pouvez consulter en ligne. 


\title{
RÉPONSES AU COMMENTAIRE DE M. ANDRÉ GOMBAY SUR LA STRUCTURE PERFORMATIVE DU LANGAGE JURIDIQUE
}

\author{
par Georges-A. Legault
}

Lorsque j'ai accepté de répondre à la critique que $\mathrm{M}$. André Gombay devait faire de mon livre La structure performative $d u$ langage juridique, j'imaginais pouvoir faire participer le lecteur à un dialogue qui aurait permis de rectifier et d'approfondir certains aspects de ma pensée en philosophie du droit. Projet intéressant et stimulant qui, malheureusement, ne s'est pas concrétisé, puisque la critique qu'on me soumet m'oblige à redire ce que j'ai déjà écrit. Étant donné que plusieurs d'entre vous n'ont pas lu ce livre, j'envisage donc de répondre point par point à la critique de $\mathrm{M}$. Gombay qui, à maints égards, semble critiquer un autre livre que le mien. Le texte même de la critique de $M$. Gombay m'oblige à répondre d'une manière que j'aurais aimé tout autre mais qui prendra la forme de la critique de la critique.

La critique, présentée par $M$. Gombay, de la première partie de mon livre regroupe en fait des commentaires tantôt allusifs, tantôt plus articulés, qui portent sur l'introduction et la première partie. Me permettant de restituer l'ordre initial du livre, je retiens comme une critique de l'introduction (et même de l'avant-propos) le point suivant. "D'après Legault, le philosophe a pour tâche de présenter un 'modèle' d'intelligibilité du droit. Cette expression - qui apparâit dès la première ligne de l'ouvrage - est loin d'être transparente; et pourtant elle ne nous est jamais bien expliquée. Legault, je crois, en revient au vieil idéal socratique, la recherche de l'essence. " ${ }^{1}$

1. Gomear, A., "Écude critique, Legault G.-A., La structure performative du langage juridique, "Philosopbiques, vol. V, numéro 2, p. 309. 
J'admets volontiers que l'expression 'modèle d'intelligibilité' n'est pas transparente. D'ailleurs peu d'expressions ont cette vertu. En effet, on peut se demander si « la recherche de l'essence », comme retour au "vieil idéal socratique », est plus transparente que 'modèle d'intelligibilité'. Ces expressions, on le voit bien, ont besoin d'être expliquées. Or, nous dit M. Gombay, «l'expression 'modèle d'intelligibilité' ne nous est jamais bien expliquée ». Je souligne le mot «bien » parce qu'il transforme un jugement de fait en jugement de valeur. L'auteur de la critique admet donc qu'il existe une explication, mais il l'évalue comme insuffisante. Or voici, malgré sa longueur, l'explication de cette expression, telle qu'on la retrouve dans mon livre.

1. L'affirmation suivante de J. Miedzianagora dans Philosophies positivistes du droit et droit positif (Paris, L.G.D.J., 1970, p. 1) doit donc être atténuée. "Ils établissent des modèles d'intelligibilité du droit tel que celui qui existe dans nos sociétés, et si parfois ces modèles nous sont présentés comme ceux de tout droit positif quelconque, en vérité ils concernent essentiellement le droit des sociétés dites «civilisés $»$ contemporaines $»$.

2. Les trois expressions, modèle d'intelligibilité, phénomène juridique, réalité juridique, présentent le vocabulaire général par lequel les données de cette étude sont circonscrites dans l'introduction. Si les deux dernières renvoient à l'objet analysé, le droit, la première réfere à notre méthode. Nous opposons ainsi la réalité juridique au phénomène juridique à cause de la tendance qui existe dans la philosophie analytique du droit à interpréter l'ensemble des activités qui réferent directement ou indirectement au droit en fonction des institutions, officielles. Certes le droit se manifeste en premier lieu par ces institutions: le Parlement et les tribunaux. Cependant, l'étude du droit exige que l'on s'arrête à sa manifestation concrète, les textes de loi et leurs relations avec les gens soumis à la loi. Ainsi, les activités les plus diverses revêtent un sens déterminé grâce aux propositions du droit. Le droit, perçu comme un langage qui s'adresse à des individus et selon lequel ceux-ci accomplissent ou n'accomplissent pas des gestes, forme donc le cœur de ce que nous dénommons la réalité juridique. Cependant, ce concept est plus englobant que celui de phénomène juridique puisque le droit conçu comme langage comprend la source d'émission - le 
Parlement - et celle d'interprétation - les tribunaux. Le terme " modèle d'intelligibilité " provient de Miedzianagora (cf. note 1) et réfere à la conceptualisation de la réalité juridique. Puisque la philosophie analytique ne prétend aucunement rejoindre l'essence métaphysique du droit, elle présente ses résultats sous forme de modèle. Ce terme s'applique davantage à notre étude puisqu'il s'agit d'employer un modèle particulier - la structure performative - pour expliquer le droit ${ }^{2}$.

Malheureusement M. Gombay n'indique pas ses critères pour dire que cette explication est insuffisante. Ne sachant pas pourquoi, à son avis, l'expression n'est « jamais bien expliquée », il m'est impossible de répondre davantage.

Quant au fait que $M$. Gombay croit que je recherche l'essence, un retour 'au vieil idéal socratique', qu'en est-il ? Après avoir évoqué les aspects multiples des lois et leur complexité, M. Gombay affirme que « il (Legault) entend une réponse unique à la question 'qu'est-ce qu'une loi ?', réponse qui vaudrait pour toute loi, quel que soit le secteur du droit auquel elle appartienne, et quel que soit le type de son rapport avec l'individu sous lequel on l'envisage ${ }^{3}$ ". Lorsqu'on compare la question de $M$. Gombay à celle que je propose dans l'introduction: "Qu'est-ce que le droit $^{4}$ ?", on s'interroge. Comment s'explique la confusion entre 'le droit' et 'une loi' ? Le texte de M. Gombay nous livre un indice. Au sujet du modèle de Kelsen, M. Gombay note entre parenthèses " (loi = norme prévoyant sanction) $)^{5}$. Or, dans le contexte de la pensée de Kelsen, il ne peut s'agir d'une loi, mais de la loi en général. Et encore, il faut bien comprendre que l'expression 'la loi' ne doit pas être utilisée pour décrire uniquement la législation. Kelsen veut faire une "théorie pure du droit». Le concept central de cette théorie est celui de norme; or ce concept ne peut se réduire au contenu d'une loi. Les questions : "qu'est-ce que le droit?", "qu'est-ce qu'une norme?", «qu'est-ce que la loi ?" (entendue comme

2. LeGault, G.-A., La structure performative du langage juridique, P.U.M., Montréal, 1977, p. 14 .

3. GOMBAY, A., art cit., p. 310.

4. Legiaul.T, C. - A., op. cit., p. 13

5. GOMBAY, A., art cit., p. 310 . 
qu'est-ce que la législation ?') et " qu'est-ce qu'une loi ?" sont certes reliées, mais elles ne sont pas identiques. Ainsi Legault n'entend pas donner une réponse unique à la question 'qu'est-ce qu'une loi ?' mais à la question 'qu'est-ce que le droit ?' C'est pourquoi, préférant aborder la question la plus générale, j'ai utilisé les expressions de réalité juridique et de phénomène juridique. En effet, le droit est, si l'on y réfléchit bien, beaucoup plus que ne l'indique $M$. Gombay lorsqu'il mentionne uniquement les aspects multiples d'une loi. Regardons notre réalité quotidienne. Combien d'expressions de notre langue ont des connotations juridiques, tels le mien, le tien? Au travail, sur la route, lors de nos achats, de la naissance à la mort, beaucoup de nos faits et gestes portent l'empreinte du droit. Mais combien d'entre eux nécessitent le recours aux institutions, (tribunaux, saisie, prison)? La proportion est faible. C'est pourquoi la description du droit ne doit pas s'intéresser uniquement aux manifestations institutionnelles, ce que j'ai appelé le phénomène juridique, et ceci au détriment de la réalité juridique.

Une fois corrigée, la critique de M. Gombay demeure-telle pertinente? Chercher une réponse à "Qu'est-ce que le droit ? » et apporter comme réponse la structure performative du droit, cela relève-t-il de l'essence du droit ? Pour répondre à cette question, telle que formulée par M. Gombay, il faudrait d'abord s'entendre sur la définition du mot « essence " et distinguer l'essence socratique de l'essence platonicienne, aristotélicienne, husserlienne ou autre. Je n'ai pas l'intention de faire ce travail inutile. Je me contente de préciser que notre droit contemporain, notre droit canadien et québécois, est organisé formellement. Telle est la conclusion de mon étude. Cette organisation formelle, que nous retrouvons avec des contenus et des modes d'opération très différents selon les institutions et les actes juridiques, ressemble à l'organisation formelle du langage. C'est ce que j'ai appelé la structure performative du langage juridique. Or, rien dans ce livre ne pose cette structure comme l'essence du droit, essence transcendante, anhistorique ou éternelle ${ }^{6}$.

6. Lecialit. G.-A., op. cit., p. 14, note 2, déjà citée. 
Commentant la première partie de mon livre consacrée à l'exposé de certaines doctrines d'inspiration positiviste, $\mathbf{M}$. Gombay précise : «Exposé cependant gouverné par une thèse. Selon notre auteur, le mouvement positiviste en philosophie du droit est poussé par une dynamique interne vers l'analyse du langage juridique; or que ce soit là son vrai destin, les membres du mouvement lui-même ne l'ont pas encore pleinement reconnu ${ }^{7}$. "Cette formulation me laisse songeur. L'auteur voulait-il seulement décrire ce que je tente de faire dans cette première partie ou avait-il une critique à formuler? Certains d'entre vous liront ce passage en retenant, d'une part, le rôle que joue le 'cependant' dans «Exposé cependant gouverné par une thèse » et, d'autre part, la division marquée par «Selon notre auteur » (l'exposition de la thèse) et «Or » (la critique). J'hésite à accepter cette interprétation non seulement parce que la critique est faible, mais aussi parce que les justifications ne prouvent rien. En effet, ces justifications se résument à ceci : Tant que les membres du mouvement positiviste n'auront pas pleinement reconnu la thèse que Legault défend (que je suis supposé défendre), cette thèse est inadmissible. Cette affirmation surprend encore plus lorsqu'on voit M. Gombay affirmer plus loin : «Qu'une analyse du langage soit ainsi l'aboutissement naturel d'une réflexion sur le droit, les membres de l'école positiviste l'ont bien aperçu ${ }^{8}$. " Certes M. Gombay puurrait soutenir qu'ils ne l'ont qu'aperçu, mais qu'ils ne l'ont pas pleinement reconnu. Or la thèse ne serait valable, selon lui, que dans le dernier cas. En fait, si la première partie de mon livre défend une thèse qui n'est pas prouvée, il devrait être facile de la critiquer. Mais voilà, défend-elle une thèse ?

Selon M. Gombay, je défends la thèse suivante : "Le mouvement positiviste en philosophie du droit est poussé par une dynamique interne vers l'analyse du langage juridique." Deux expressions captent ici toute mon attention : celle de 'mouvement positiviste' et celle 'd'être poussé'. Ces expressions suggèrent que je conçois le positivisme en philosophie du droit comme une organisation, une école de pensée déjà structurée $a$

7. GOMBAY, A., art cit., p. 309-310.

8. Gombay, A., art. cit., p. 311. 
priori et que ce mouvement est contraint de s'épanouir d'après la logique interne de sa structure. C'est pourquoi le mouvement serait poussé par une dynamique interne. Or je ne défends cette thèse ni dans la première partie ni ailleurs ${ }^{9}$. Au contraire, comme je l'indique dans l'avant-propos et comme je le précise dans l'introduction, la première partie vise deux objectifs : présenter les résultats des analyses antérieures, mais de façon critique afin de situer les problèmes à résoudre par la structure performative $^{10}$.

Ainsi M. Gombay ne dit rien ou presque rien sur le développement de la première partie. Après un commentaire sur Kelsen, il présente les raisons qui, selon lui, me conduisent à utiliser un modèle issu de la philosophie du langage. Sur ma critique de Kelsen, il dit : "Ainsi le 'modèle' proposé par Kelsen (loi $=$ norme prévoyant sanction) s'avère - selon Legault - défectueux parce qu'il implique un primat du pénal par rapport au civil . . . ${ }^{11}$ » Encore une fois, le raisonnement de M. Gombay est faux. En effet, il confond les caractéristiques du modèle kelsennien avec les raisons de son insuffisance. Le modèle de Kelsen est rejeté parce qu'entre autres, son concept de norme ne peut pas rendre compte des textes de la $\operatorname{loi}^{12}$.

Les derniers commentáires de M. Gombay sur cette première partie ne sont pas de meilleure qualité. Les affirmations suivantes sont tellement erronées qu'il semble que l'auteur critique un autre livre que le mien. "Mais ce qui amène vraiment la théorie positiviste du droit à devenir une philosophie du langage juridique, c'est le problème de la validité $^{13}$." «Toutefois, selon Legault, ces analyses - même si elles sont justes - laissent sans réponse le problème fondamental : ces normes, ces règles, pourquoi déterminentelles la conduite de ceux qui les observent ${ }^{14}$ ? "Il suffit de lire la première partie du livre pour comprendre que le problème de la validité, important pour Kelsen, n'est plus une préoccu-

9. J'affirme le contraire de cette thèse à la page 191.

10. LeGalili, G.-A., op. cit., p. 27.

11. Gomisay, A., art. cit., p. 310.

12. Leciaulit, G.-A., op. cit., p. 40, 46, 79, 125, 191 et d'autres.

13. GOMBAY, A., art. cit., p. 310.

14. Gombay, A., art. cir., p. 310-311. 
pation première pour Ross et d'autres auteurs. Dois-je préciser et souligner que le problème de la validité du droit n'est pas le problème fondamental de ce livre, pas plus qu'il n'explique pourquoi la philosophie du droit s'oriente vers une philosophie du langage? De plus, la lecture de cette partie montre clairement que les modèles d'intelligibilité présentés par Kelsen, Ross, Olivecrona, Hedenius et Hart sont insuffisants. Comment puis-je alors les trouver justes? Il ne faut pas se méprendre. Ces théories ne sont pas insuffisantes parce qu'elles ne donnent pas de réponse à la question de la validité, comme le prétend M. Gombay. Elles sont insuffisantes parce que, d'une part, ces auteurs ne peuvent expliquer le langage juridique, celui des codes civil et pénal, et parce que, d'autre part, ils ne peuvent rendre compte de l'utilisation de formules juridiques par les tribunaux et les citoyens au niveau des actes juridiques. Ainsi, ce qui échappe à l'analyse de ces auteurs, c'est le langage juridique et son application.

L'objet même de notre étude, le droit, qui se concrétise dans des textes, exige qu'on passe par l'analyse du langage. Voilà pourquoi un modèle issu de l'analyse du langage peut nous être utile. En effet, comment expliquer le langage des lois sans une théorie du langage ? Comment expliquer les actes juridiques tels le jugement, le contrat, la législation sans prendre en considération le rôle de l'émetteur, celui du receveur et celui du langage? Le lecteur retrouve toutes ces raisons aux pages 157 et 158 de mon livre. Malheureusement, celui qui n'a pas lu ces pages et qui retient la citation ${ }^{15}$ que M. Gombay présente comme étant la seule justification de ma part pour utiliser un modèle issu de l'analyse du langage, celui-là est induit en erreur.

Les commentaires de M. Gombay sur la deuxième partie du livre sont très rares. Après un bref résumé de la problématique de la philosophie du langage, il affirme: "Legault consacre de longues pages à une discussion, souvent très minutieuse, de ces questions et de la question plus générale de ce qui est requis pour la réussite d'un acte de parole $^{16}$. " À ceux qui ne sont pas initiés à la philosophie

15. Gomlis, A., art cit., p. 311.

16. Gomsis, A., art cit., p. 312. 
du langage et à la manière qu'ont les philosophes du langage d'étudier la promesse par exemple, je conseille de lire J.L. Austin et particulièrement $\mathrm{J}$. Searle. Vous constaterez alors que non seulement l'étude minutieuse de ces auteurs exige un vocabulaire et un mode d'expression particuliers, mais encore que la transformation de ces interprétations oblige un auteur à discuter sur le même terrain, avec les mêmes expressions. Ainsi, ce qui apparaît à $M$. Gombay comme du " charabia " ${ }^{17}$ (notez bien qu'il cite alors un passage provenant de la deuxième partie et qu'il le présente hors de tout contexte) s'avère une réponse à la classification même de J. Searle. Certes on peut critiquer les philosophes du langage et évaluer que leur mode d'expression symbolique $\mathrm{E}$ et $\mathrm{R}$, que les expressions "E croit $\mathrm{p}$ », " $\mathrm{E}$ croit a dans l'intérêt de $\mathrm{R}$ », etc., ne sont pas justifiables. Mais pour faire une telle critique, il ne suffit pas de citer un texte et de laisser au lecteur la tâche de trouver les critères d'évaluation à partir de cette seule citation. Cette manière de procéder ne constitue ni une preuve empirique ni une forme d'argumentation rationnelle, mais elle relève plutôt du style publicitaire et de la démagogie.

Nous arrivons maintenant aux commentaires sur la troisième partie de mon livre, «la plus intéressante de l'ouvrage $^{18}$ » affirme $M$. Gombay, sans qu'on puisse savoir pourquoi il la juge ainsi. Évidemment, la troisième partie est la plus cruciale, car c'est le moment de vérité. Alors que la première partie a situé les principales difficultés rencontrées par les théories antérieures, difficultés que les auteurs n'ont pas surmontées, et que la deuxième partie a développé un modèle de structure performative, il s'agit de savoir maintenant si cette structure performative peut être utile pour décrire le droit. Qu'en pense M. Gombay? «Donc le livre de Legault est souvent instructif. Cependant il n'est pas une réussite. Pourquoi ? Tout d'abord et centralement, Legault n'établit pas ce qu'il pense avoir établi ${ }^{19}$. " Le raisonnement de $M$. Gombay, raisonnement qui fonde ce jugement catégorique "Legault ne l'établit en rien ${ }^{20}$ ", s'articule ainsi. Après la

17. Gommsy, A., art cit, p. 316.

18. Gomlir, A., art cit., p. 312.

19. Gomlay, A., art cit., p. 313.

20. Gombay, A., art cit., p. 313. 
description de mon prétendu modèle, suivent des commentaires sur l'impossibilité inhérente au projet. Ensuite à titre d'exemple, M. Gombay nous parle de l'analyse du faux prétexte, du meurtre, de l'homicide involontaire et de la punition. Ces exemples prouvent, selon lui, pourquoi le 'dessein' proposé par l'auteur n'est pas réussi. Suivons les étapes de ce raisonnement.

Selon M. Gombay, mon modèle d'intelligibilité du droit consiste à "démontrer que les mêmes conditions déterminent réussite et ratage d'un acte de parole (tel que la promesse) et réussite et ratage de tout acte proprement juridique ${ }^{21} »$. Deux expressions attirent cette fois mon attention. Le mot 'même' que M. Gombay souligne dans l'expression "les mêmes conditions» et l'expression «d'un acte de parole». Notez bien, cette fois, que je souligne le un. S'agit-il là de ma thèse ? Vous l'aurez sans doute deviné : NON. C'est pourquoi M. Gombay a tant de facilité à montrer l'impossibilité inhérente à ce projet qu'il vient d'inventer. Il a donc raison de dire: «Comment y parviendrait-il ? Ce n'est pas en dégageant l'affinité entre certains actes de caractère juridique et la promesse qu'on arriverait à démontrer une vérité sur le droit, ou même le langage juridique, pris dans son ensemble ${ }^{22}$." Mais je ne prétends pas que l'analyse de la promesse et des conditions qui déterminent la réussite ou le ratage de la promesse (un acte de parole) puisse servir à expliquer l'ensemble du droit. Au contraire, la promesse, la demande, le commandement ne sont que quelques actes de discours. Leurs conditions de réussite ne sont pas les mêmes, puisque les actes ne sont pas identiques. Par contre, chaque acte de discours est constitué d'une règle fondamentale performative, de règles de compétence, de règles d'objet et de règles de communication. Voilà ce que j'ai appelé la structure performative. Dans la troisième partie je procède donc à l'analyse des différents actes juridiques et je montre que ces actes comme les actes de discours sont organisés d'après les règles que je viens de mentionner. Or, de cela M. Gombay ne dit rien. Ce n'est pas la première fois que $M$. Gombay prend la partie pour le tout.

21. GoMl3h, A., art cit, P. 313.

22. Gostsiy, A., art. cit., p. 313-314. 
Déjà dans sa critique de l'introduction il avait confondu «qu'est-ce que le droit? » avec « qu'est-ce qu'une loi ? » Ici, il confond les conditions d'un acte de parole (la promesse) et les conditions générales de tout acte de discours.

La troisième partie de mon livre montre donc comment la structure performative, telle que définie dans la deuxième partie, explique à la fois les phénomènes juridiques tels la législation, le jugement, la constitution et la réalité juridique, les faits et gestes des gens soumis au droit. Je n'ai pas l'intention de reprendre cette troisième partie, mais je désire préciser l'argumentation que i'y présente. D'une part, il ne faut pas oublier qu'un acte juridique peut s'interpréter soit comme un acte institutionnel, soit comme un acte posé par des individus. D'autre part, il ne faut pas oublier que, pour nombre d'auteurs, le droit civil et le droit pénal ne peuvent se comparer si ce n'est par le biais de la sanction. L'argumentation de la troisième partie tient compte de ces deux points. Elle commence par l'analyse du contrat afin de démontrer la structure performative appliquée par les individus. Les liens entre le contrat et la promesse font qu'il est facile de montrer non seulement la structure performative du contrat, mais encore que les mêmes conditions s'appliquent au contrat et à la promesse. Ainsi, la structure performative de certains actes juridiques est presque identique, au niveau du contenu des règles, à celle de certains actes de discours. Afin de démontrer que la parole n'est pas nécessaire en droit civil pour réaliser un performatif, j'analyse donc l'acte juridique qu'est la prescription. Le même raisonnement qui a guidé l'analyse de ces actes en droit civil s'applique pour l'analyse du droit pénal. D'abord, il y a l'étude d'une situation où la parole est nécessaire: le faux prétexte, ensuite, celle de situations où elle ne l'est pas, le vol, le meurtre. L'analyse de la responsabilité civile et pénale démontre de nouveau comment les deux droits peuvent se comparer, ce qui n'était pas admis auparavant. Or cette comparaison peut se faire grâce à la structure performative de ces actes juridiques.

Or, M. Gombay affirme : "Il est symptomatique par exemple que le délit criminel que Legault étudie de plus près soit le faux prétexte. Bien sûr, le faux prétexte s'apparente à la 
fausse promesse ; mais est-ce un délit typique ${ }^{23}$ ? "Comme je viens de l'expliquer, l'étude du 'faux prétexte' n'est pas le symptôme d'une maladie, d'une erreur d'argumentation. Au contraire, son analyse montre que, même en droit pénal, il existe des délits où la parole est nécessaire. Ainsi le droit civil et le droit pénal ne sont pas si différents qu'on le pense. De plus, je n'ai pas choisi le faux prétexte parce qu'il s'apparente à la fausse promesse, car depuis quand une promesse est-elle fausse? Si on veut comparer le faux prétexte à un acte de discours, il faut le comparer à l'affirmation fausse, non à la promesse. L'interrogation de M. Gombay, « est-ce un délit typique? " en parlant du faux prétexte, suggère la critique suivante. On ne peut pas généraliser les conclusions de l'étude du faux prétexte aux autres délits criminels. Or cette critique de M. Gombay serait justifiée, selon lui, par mon analyse du meurtre, de l'homicide involontaire et de la punition. Qu'en est-il ?

L'analyse des actes juridiques relevant du droit pénal a été amorcée par l'étude du faux prétexte. J'ai expliqué plus haut les raisons qui motivent ce choix. Il ne faut pas oublier que le faux prétexte est un délit en droit pénal qui ressemble au vol. En fait, obtenir par faux prétexte quelque chose, c'est une manière plus subtile de voler. C'est pourquoi, compte tenu de ce qui fut démontré par l'analyse de la prescription en droit civil, compte tenu également de l'analyse du faux prétexte, il est possible de généraliser l'application de la structure performative aux autres délits tels le vol, le meurtre et l'homicide involontaire. Or, la structure performative, comme je l'ai précisé, comprend une règle fondamentale performative, des règles de capacité, des règles d'objet et des règles de communication. Omettant de dire quoi que ce soit sur les autres règles des actes juridiques, $M$. Gombay affirme: « Ainsi il (Legault) affirme sans broncher (414) que le meurtre est un acte de communication réussi, alors que l'homicide involontaire est un acte de communication raté. Vraiment? Ou bien, page 504, Legault croit établir - encore une fois à partir de sa notion de communication - que la réhabilitation

23. GomksY, A., art cit., p. 314. 
du coupable est le seul but valable de la punition ${ }^{24}$." M. Gombay consacre donc son attention à la notion de communication, alors que je parle des règles d'un performatif et de certaines règles relatives à la communication. Encore une fois, M. Gombay transforme la partie en tout. Or, en droit pénal, l'intention joue un rôle important, puisque l'intention est transmise lors du délit. De plus, il n'y a pas de délit sans intention. C'est pourquoi j'affirme sans broncher à la page 414: "Qu'il s'agisse du vol, du meurtre, de l'homicide involontaire, etc., ces actes rejoignent directement autrui, dans son corps ou dans ses biens. C'est pourquoi l'accomplissement des gestes revêt aussi la signification d'un acte de communication. En effet, lorsque le tribunal déclare l'individu coupable, il interprète les gestes accomplis comme un acte de communication véhiculant une intention malveillante à l'égard d'un autre."

Au sujet de la dernière remarque de M. Gombay, sur la punition comme acte de communication, commentaire qui provient de mes remarques à la page 504 , je dois avouer que je n'y comprends rien. Certes je parle bien de la punition à cet endroit, mais il n'y a aucune allusion à un modèle de la communication. Il ne faut pas oublier aussi que M. Gombay utilise ici des remarques de la conclusion qu'il confond avec l'argumentation de la troisième partie. De plus, je ne cherche nullement à ébranler le champion de la punition expiatoire ; au contraire, je constate, à partir de l'analyse du droit pénal, que le droit cherche effectivement à faire expier quelqu'un. C'est pourquoi j'affirme à la page 504 :

«Pourquoi incarcérer un individu pour un de ses actes? La peine, l'emprisonnement, deviennent des moyens symboliques de réparation, puiqu'il ne s'agit pas d'une réparation matérielle au sens ou les victimes de l'infraction recevraient quelque chose de matériel comme les victimes de la responsabilité civile. Cependant, l'incarcération demeure réparatrice, car il s'agit avant tout d'expier une faute individuelle. »

Comment M. Gombay a-t-il pu lire autrement ce passage ?

L'analyse critique de $M$. Gombay se termine avec

24. GомваY, A., art. cit., p. 314 
plusieurs commentaires sur la qualité de l'expression écrite du livre. J'avoue sincèrement que dans les 522 pages du livre, il y a sans doute des lacunes qui y nuisent. Cependant, j'aimerais souligner un point concernant la conclusion finale de $\mathbf{M}$. Gombay. La question "Comment une maison de presse universitaire a-t-elle pu permettre la parution d'un tel texte? " peut avoir deux significations. D'une part, le texte de l'auteur aurait dû être refusé ; d'autre part, la maison de presse universitaire aurait dû soigner davantage la qualité du texte. À quoi servent tous ces comités de lecture et de révision, sinon à donner au public un livre de meilleure qualité possible? Mais dans certains cas, certaines personnes ont la responsabilité d'intervenir pour améliorer la qualité d'un manuscrit avant qu'il soit soumis à une maison d'édition. Je songe ici particulièrement aux manuscrits qui, comme le mien, sont des thèses de doctorat. À quoi sert donc le jury? Ne doit-il pas suggérer des corrections mineures ou majeures si c'est nécessaire ? Or, M. Gombay était un des membres du jury qui a évalué ma thèse. Jamais il ne m'a remis cette liste d'erreurs que jaurais pu corriger, alors que je la lui ai demandée verbalement après la soutenance et par écrit ensuite.

Certes, M. Gombay peut se demander : "Comment une maison de presse universitaire a-t-elle pu permettre la parution d'un tel texte? "De mon côté je me demande : "Comment la revue Pbilosophiques peut-elle publier comme Étude critique un texte tel que celui de M. Gombay et obliger (car qui ne dit mot, consent) un auteur à répondre dans un nombre limité de pages à une critique qui est loin de faire avancer la discussion philosophique et la qualité de la réflexion en philosophie?"

Coilège Bois de Boulogne. 